\title{
A convolution integral equation solved by Laplace transformations
}

N.M. TEMME

Department of Applied Mathematics, Centre for Mathematics and Computer Science, 1098 SJ Amsterdam, The Netherlands

Received 23 July 1984

Revised 16 October 1984

$\boldsymbol{A}$ bstract: We consider the integral equation

$P(t)=\int_{0}^{t} K(t-\tau) p(\tau) \mathrm{d} \tau+r(t)$

where both $K(t)$ and $r(t)$ behave as $\exp \left(\alpha t^{3}\right)$ as $t \rightarrow \infty(\alpha>0)$. So straightforward application of the Laplace transform technique is not possible. By introducing a complex parameter the equation is solved in the complex domain. Analytic continuation with respect to this parameter yields the desired solution. For a particular example (which arose in a statistical problem on estimating monotone densities) we describe the construction of the explicit solution of the equation.

Keywords: Integral equation of convolution type, Laplace transformations, analytic continuation, Airy functions.

\section{Introduction}

We are interested in the solution $p_{0}$ of the integral equation

$$
p_{0}(t)=(2 \pi)^{-1 / 2} \int_{0}^{t} p_{0}(y) \mathrm{e}^{-(1 / 2) t y(t-y)}(t-y)^{1 / 2} \mathrm{~d} y+r_{0}(t)
$$

where $t>0$ and

$$
r_{0}(t)=-\left(\frac{1}{2} \pi\right)^{1 / 2}+t^{3 / 2}-(2 \pi)^{-1 / 2} \int_{0}^{t}(t-y)^{1 / 2} y^{-3 / 2}\left[1-\mathrm{e}^{-(1 / 2) t y(t-y)}\right] \mathrm{d} y .
$$

This equation arose in a statistical problem on the estimation of monotone densities considered in Groeneboom [1].

First we give an expansion of $p_{0}(t)$ in powers of $t$, yielding a good approximation for small and intermediate values of $t$. Our main concern is an expansion at infinity. We obtain a convergent expansion which can be used for all positive values of $t$.

\section{Expansion at $t=0$ and at infinity}

The function $r_{0}(t)-t^{3 / 2}$ is an entire function; this follows immediately from (2). Substituting the Taylor expansion of $r_{0}(t)-t^{3 / 2}$ into (1) we obtain

$$
p_{0}(t)=-\left(\frac{1}{2} \pi\right)^{1 / 2} \sum_{n=0}^{\infty} \alpha_{n} t^{3 n}+t^{-3 / 2} \sum_{n=1}^{\infty} \beta_{n} t^{3 n}
$$


with $\alpha_{0}=1, \alpha_{1}=\frac{7}{48}, \beta_{1}=\frac{2}{3}, \beta_{2}=\frac{4}{189}$. It follows that both series in (3) represent entire functions of $t$. Representation (3) was useful in obtaining numerical information on $p_{0}(t)$ for small and intermediate values of $t$. Higher coefficients $\alpha_{n}, \beta_{n}$ can be computed by recursion.

By substituting an asymptotic expansion of $r_{0}(t)$ into (1) we obtain for $p_{0}(t)$ the expansion $p_{0}(t) \sim-t^{-3 / 2}, \quad t \rightarrow \infty$

All remaining coefficients of $t$-powers seem to vanish. So we obtained the conjecture that $p_{0}(t)=-t^{-3 / 2}+q(t)$ with $q(t)=\mathcal{O}\left(t^{-n}\right)$ as $t \rightarrow \infty$, for any $n$. For the statistical problem this information on $p_{0}$ and $q$ was not sufficient and it appeared that it was rather difficult to obtain the asymptotic behaviour of $q$ directly from (1).

\section{A convolution equation}

When we write $-\frac{1}{2} t y(t-y)=\frac{1}{6}(t-y)^{3}+\frac{1}{6} y^{3}-\frac{1}{6} t^{3}$ we obtain for (1)

$$
p_{0}(t) \mathrm{e}^{(1 / 6) t^{3}}=(2 \pi)^{-1 / 2} \int_{0}^{t} p_{0}(y) \mathrm{e}^{(1 / 6) y^{3}}(t-y)^{1 / 2} \mathrm{e}^{(1 / 6)(t-y)^{3}} \mathrm{~d} y+\mathrm{e}^{(1 / 6) t^{3}} r_{0}(t) .
$$

Introducing the functions

$$
p_{1}(t)=p_{0}(t) \mathrm{e}^{(1 / 6) t^{3}}, \quad r_{1}(t)=r_{0}(t) \mathrm{e}^{(1 / 6) t^{3}}, \quad K_{1}(t)=(2 \pi)^{-1 / 2} t^{1 / 2} \mathrm{e}^{(1 / 6) t^{3}},
$$

we have the convolution type equation

$$
p_{1}(t)=\int_{0}^{t} p_{1}(y) K_{1}(t-y) \mathrm{d} y+r_{1}(t) .
$$

A usual approach for such linear equations is the Laplace transform technique. It is readily seen that the Laplace transform of $K_{1}(t)$ is not defined. The same is true for $p_{1}(t), r_{1}(t)$.

To avoid this complication we consider the modified equation

$$
p(t)=\int_{0}^{t} p(y) K(t-y) \mathrm{d} y+r(t)
$$

where

$$
p(t)=p_{1}(\lambda t), \quad r(t)=r_{1}(\lambda t), \quad K(t)=\lambda K_{1}(\lambda t),
$$

with $\lambda \in S=\{\lambda \in \mathbb{C} \mid \lambda \neq 0,-\pi<\arg \lambda<\pi\}$. Then (7) has to be solved for $\lambda=1, t>0$.

Remark. Equation (7) can be solved by constructing a series expansion as in (3) or by the Neumann-series of iterated kernels. For each $t>0$, the terms in these expressions are analytic functions of $\lambda, \lambda \in S$. The series converge uniformly with respect to $\lambda$ in compact sets of $S$. Hence, $p(t)$ is analytic in any finite point $\lambda \in S$.

\section{Laplace transform techniques}

We continue the analysis for $\lambda \in \Lambda$, where

$$
\Lambda=\left\{\lambda \in \mathbb{C} \mid \lambda \neq 0, \frac{1}{6} \pi<\arg \lambda<\frac{1}{2} \pi\right\} .
$$


Then the Laplace transform of

$$
K(t)=(2 \pi)^{-1 / 2} \lambda(\lambda t)^{1 / 2} \mathrm{e}^{(1 / 6) \lambda^{3} t^{3}}
$$

exists, i.e., we can define

$$
\hat{K}(s)=\int_{0}^{\infty} \mathrm{e}^{-s t} K(t) \mathrm{d} t, \quad \lambda \in \Lambda
$$

and $\hat{K}(s)$ is an entire function of $s$. Some asymptotic analysis is needed to show that $r(t)=\mathcal{O}\left[t^{\alpha} \exp \left(\frac{1}{24} \lambda^{3} t^{3}\right)\right]$ as $t \rightarrow \infty, \lambda \in \Lambda$, where $\alpha$ is some real number. Hence $\hat{r}(s)$ exists as well $(\lambda \in \Lambda)$ and is an entire function of $s$.

Using the convolution rule for Laplace transformations we obtain for the solution of (7)

$$
p(t)=\frac{1}{2 \pi \mathrm{i}} \int_{c-\mathrm{i} \infty}^{c+\mathrm{i} \infty} \mathrm{e}^{s t} \hat{p}(s) \mathrm{d} s, \quad \lambda \in \Lambda
$$

where

$$
\hat{p}(s)=\hat{r}(s) /(1-\hat{K}(s))
$$

and where $c$ should be large enough to locate all poles of the meromorphic function $\hat{p}(s)$ to the left of the path of integration.

To compute $\hat{r}(s)$ it is convenient to introduce

$$
\phi_{\alpha}(t)=(\lambda t)^{\alpha} \mathrm{e}^{(1 / 6) \lambda^{3} t^{3}}, \quad \hat{\phi}_{\alpha}(s)=\int_{0}^{\infty} \mathrm{e}^{-s t} \phi_{\alpha}(t) \mathrm{d} t
$$

where in the integral $\operatorname{Re} \alpha>-1, \lambda \in \Lambda$. The function $r(t)$ can be written in the form

$$
r(t)=\phi_{3 / 2}(t)+(2 / \pi)^{1 / 2} \lambda^{2} t\left[\phi_{1 / 2} * \phi_{1 / 2}\right](t)-\frac{1}{2}(2 \pi)^{-1 / 2} \lambda\left(2+\lambda^{3} t^{3}\right)\left[\phi_{-1 / 2} * \phi_{-1 / 2}\right](t),
$$

where * denotes the convolution operator. Using

$$
\left\{\begin{array}{l}
\frac{\mathrm{d}}{\mathrm{d} s} \hat{\phi}_{\alpha}(s)=-\lambda^{-1} \hat{\phi}_{\alpha+1}(s), \\
(\alpha+1) \hat{\phi}_{\alpha}(s)=s \lambda^{-1} \hat{\phi}_{\alpha+1}(s)-\frac{1}{2} \hat{\phi}_{\alpha+3}(s)=-s \hat{\phi}_{\alpha}^{\prime}(s)+\frac{1}{2} \lambda^{3} \hat{\phi}_{\alpha}^{\prime \prime \prime}(s)
\end{array}\right.
$$

where the primes denote derivatives with respect to $s$. After elementary calculations we obtain

$$
\hat{r}(s)=\hat{\phi}_{3 / 2}(s)-(2 \pi)^{-1 / 2} \hat{\phi}_{1 / 2}(s)\left[\lambda \hat{\phi}_{3 / 2}(s)-2 s \hat{\phi}_{-1 / 2}(s)\right] .
$$

Taking into account the relation $\hat{K}(s)=(2 \pi)^{-1 / 2} \lambda \hat{\phi}_{1 / 2}(s)$, see (9) and (13), we can write (12) as

$$
\hat{p}(s)=-\hat{\phi}_{-3 / 2}(s)+2 \frac{\hat{\phi}_{3 / 2}(s)-s \lambda^{-1} \hat{\phi}_{-1 / 2}(s)}{1-\hat{K}(s)} .
$$

Remark. $\hat{\phi}_{-3 / 2}(s)$ is defined by means of the recursion in (15) and not by the integral in (13). However it is easily verified that for any $c, t>0, \lambda \in \Lambda$

$$
\frac{1}{2 \pi \mathrm{i}} \int_{c-\mathrm{i} \infty}^{c+\mathrm{i} \infty} \mathrm{e}^{s t} \hat{\phi}_{-3 / 2}(s) \mathrm{d} s=\phi_{-3 / 2}(t) .
$$




\section{Airy functions}

A remarkable simplification of (16) is possible by using Airy functions. For properties of these functions we refer to Olver [2]. Starting with the well-known differential equation $\ddot{y}-z y=0$, we can easily show that $w^{(3)}-4 z \dot{w}-2 w=0$ is solved by a product of Airy functions. For $\alpha=-\frac{1}{2}$ the third order differential equation in (15) can be reduced to the above $w$-equation. By comparing Taylor series of $\hat{\boldsymbol{\phi}}_{-1 / 2}(s)$ and the Airy functions we obtain

$$
\hat{\phi}_{-1 / 2}(s)=2^{5 / 6} \pi^{3 / 2} \lambda^{-1} A i(z)[B i(z)+\mathrm{i} A i(z)], \quad z=2^{-1 / 3} \lambda^{-1} s .
$$

By using the Wronskian $\pi^{-1}=A i(z) \dot{B} i(z)-\dot{A} i(z) B i(z)$ and by expressing $\hat{\phi}_{1 / 2}(s)$ in terms of Airy functions, it follows that

$$
1-\hat{K}(s)=2 \pi \mathrm{i} A i(z)\{\dot{A} i(z)-\mathrm{i} \dot{B} i(z)\} .
$$

Furthermore it follows from the recursions for $\hat{\phi}_{\alpha}(s)$

$$
\hat{\phi}_{3 / 2}(s)-s \lambda^{-1} \hat{\phi}_{-1 / 2}(s)=\mathrm{i} 2^{7 / 6} \pi^{3 / 2} \lambda^{-1} \dot{A} i(z)\{\dot{A} i(z)-\mathrm{i} \dot{B} i(z)\} \text {. }
$$

After these preparations the function $\hat{p}(s)$ of $(16)$ can be written as

$$
\hat{p}(s)=-\hat{\phi}_{-3 / 2}(s)+2^{7 / 6} \pi^{1 / 2} \lambda^{-1} \dot{A} i(z) / A i(z), \quad z=2^{-1 / 3} \lambda^{-1} s .
$$

With (11) and (17) we obtain

$$
p(t)=-\phi_{-3 / 2}(t)+\frac{2^{7 / 6} \pi^{1 / 2} \lambda^{-1}}{2 \pi \mathrm{i}} \int_{c-\mathrm{i} \infty}^{c+\mathrm{i} \infty} \mathrm{e}^{s t} \frac{\dot{A} i(z)}{A i(z)} \mathrm{d} s,
$$

where $c>0, \lambda \in \Lambda, t>0$. The Airy function $A i(z)$ has an infinite number of negative zeros $a_{1}$, $a_{2}, \ldots$ These zeros are simple and there are no other zeros (Olver [2, p. 414]). The poles of the integrand in (19) occur at $s_{n}=2^{1 / 3} \lambda a_{n}(n=1,2, \ldots)$, which for $\lambda \in \Lambda$ are located in the half plane $\operatorname{Re} s<0$. When we shift the contour of integration of (20) to the left we obtain a series of residues. It easily follows from asymptotic properties of the Airy functions and of the zeros that the infinite series of residues converges, while the integral tends to zero as the contour is shifted progressively to the left. So we obtain the representation

$$
p(t)=-\phi_{-3 / 2}(t)+2(2 \pi)^{1 / 2} \sum_{n=1}^{\infty} \mathrm{e}^{s_{n} t}, \quad t>0 .
$$

We now drop the condition $\lambda \in \Lambda$. The above series represents an analytic function of $\lambda$ for the domain $\operatorname{Re} \lambda>0$, while the solution $p(t)$ is obtained for $\lambda \in \Lambda$. Also, $\phi_{-3 / 2}(t)$ is an analytic function of $\lambda$ in $\operatorname{Re} \lambda>0$. From the Remark in Section 3 it follows that $p$ itself is analytic in $\operatorname{Re} \lambda>0$. By using the principle of analytic continuation it now follows that in (21) we can take $\lambda=1$. In this case we have $p(t)=p_{1}(t)=p_{0}(t) \exp \left(\frac{1}{6} t^{3}\right)$. Thus we have for the solution of (1)

$$
p_{0}(t)=-t^{-3 / 2}+2(2 \pi)^{1 / 2} \mathrm{e}^{-(1 / 6) t^{3}} \sum_{n=1}^{\infty} \mathrm{e}^{2^{1 / 3} a_{n} t}, \quad t>0 \text {. }
$$

\section{Concluding remarks}

(1) We have solved (1) for the special case (2). With this function just one series of poles and residues occurs in (20), (21). A general function $r_{0}$ will not divide out the term $\dot{A} i(z)-\mathrm{i} \dot{B} i(z)$ in 
(18). This function vanishes at the zeros of $\dot{A} i\left(z \exp \left(\frac{2}{3} \pi \mathrm{i}\right)\right)($ Olver [2, p. 414]). In the $s$-plane the zeros of this function occur at $s_{n}^{\prime}=2^{1 / 3} \lambda a_{n}^{\prime} \exp \left(-\frac{2}{3} \pi \mathrm{i}\right)$, where $a_{n}^{\prime}$ are the negative zeros of $\dot{A} i(z)$. So, $\arg s_{n}^{\prime}=\frac{1}{3} \pi+\arg \lambda$. For $\lambda \in \Lambda$ we have $\operatorname{Re} s_{n}^{\prime}<0$. In that case two series of residues appear in the solution. However, when $\lambda$ leaves the sector $\Lambda$ at $\arg \lambda=\frac{1}{6} \pi$, the zeros $s_{n}^{\prime}$ enter the half plane $\operatorname{Re} s>0$. So, when the poles $s_{n}^{\prime}$ are not cancelled by the zeros of $\hat{r}(s)$, a representation in the form of two residue series is only possible when $\lambda \in \Lambda$, but not when $\arg \lambda<\frac{1}{6} \pi$. Consequently, the analytic continuation of the results from $\lambda \in \Lambda$ to $\lambda=1$ will be much more complicated in the general case than in the case of $r_{0}$ as given in (2).

(2) In (7) and (8) we have introduced the complex parameter $\lambda$. A similar approach is possible by making $t$ complex and solving (6) for $t \in \Lambda$. The Laplace transform technique has to be modified then. To avoid this we have chosen the $\lambda$-approach.

\section{Acknowledgments}

This research is done in close collaboration with dr. P. Groeneboom of the department of Mathematical Statistics of the CWI. A joint paper will give detailed information on the underlying statistical problem and more details on the analytical elaborations.

\section{References}

[1] P. Groeneboom, Estimating a monotone density, Report MS-R8403, CWI Amsterdam, 1984; and, in: L. LeCam, Ed., Proc. Neyman-Kiefer Conference, Berkeley, June-July, 1983.

[2] F.W.J. Olver, Asymptotics and Special Functions (Academic Press, New York, 1974). 